\title{
Conjunto de Charilla, un nuevo estudio
}

\author{
Ana Belén Haro Gutiérrez*
}

\begin{abstract}
RESUMEN
El hallazgo de Charilla (Jaén), fechado por material numismático como califal es uno de los pocos conjuntos de orfebrería andalusí perteneciente a dicha época. El hecho de que aparezcan joyas junto con monedas no es algo excepcional, pero sí muy importante a la hora de fechar otros conjuntos que aparecieron sin monedas, como el de Garrucha, las piezas sueltas pertenecientes al Museo Arqueológico Nacional y el de The Walter Art Gallery (E.E.U.U.), aunque éste último está pendiente de ser revisado, ya que por técnica y estilo difiere del resto.
\end{abstract}

PALABRAS CLAVE: Al-Andalus, Califato, Numismática, Joyería, Hallazgos.

Las primeras piezas encontradas del conjunto de Charilla fueron halladas de forma casual el día I I de enero de 1977 por Alejandro López López, Rafael Gallego López y Miguel Pareja Extremera, tres alumnos de E.G.B. del Colegio Nacional Comarcal $n^{\circ} 2$ en la Aldea de Charilla, perteneciente al término municipal del Alcalá la Real (Jaén).

Días después, el profesor de los alumnos D. Manuel Gómez Palomares relataba en un informe ' que fue enviado al Director General del Patrimonio artístico y Cultural lo siguiente: “... en la mañana del día doce cuando me disponía a

\begin{abstract}
Charilla's hoard (Jaén, Spain), dated by coins as of caliphal period, is one of the few jewelry hoards well dated. The presence of coins together with jewels is not anomale but give us the possibility to date other material without coins by stilistical analysis like the hoards of Garrucha, isolated pieces from Archaeological Museum of Madrid and the materials from the The Walter Art Gallery (E.E.U.U.), very diferent in technique and style that would be revised in future.
\end{abstract}

KEY WORDS: Al-Andalus, Caliphate, Numismatic, Jewelry, Hoards.

dar comienzo a mis tareas escolares, en ese momento, se me acercaron dos alumnos, Alejandro López López y Rafael Gallego López, mostrándome tres objetos, uno de ellos era una sortija con una inscripción de letras, que yo, consideré árabes, otro de los objetos, en forma de media luna y repujado el otro objeto tenía forma de pendiente. Una vez observados los mencionados objetos pregunté cómo y donde se había encontrado; y los alumnos me relataron lo siguiente: encontrándose la tarde anterior jugando, con los otros amigos, en una excavación realizada por una máquina, detrás de la cooperativa, al coger un puñado de tierra, uno de ellos, concretamente Alejan-

\footnotetext{
* C/Huesca n¹9 5D, 28934 Móstoles (Madrid).

I Archivo del Museo Provincial de Jaén.
} 
dro López López, observó que entre ella brillaba un objeto, entonces llamó a sus amigos y aunque ellos no sabían lo que era se pusieron a escarbar en la tierra, encontrándose diversos objetos, entre ellos algo parecido a una corona. Yo, rápidamente, pensé que podría ser una tumba conteniendo el ajuar ya citado. No quise darle importancia para que ellos no se alarmaran. A las cinco, una vez finalizadas las tareas escolares, me marché con ellos a 'Charilla', fui a sus respectivos domicilios y me entregaran los objetos que habían encontrado, rápidamente fuimos al lugar del 'hallazgo', provistos de unos pequeños almocafres para remover la tierra, así lo hicimos y entonces encontramos varios objetos; después me volví a Alcalá, me presenté en el Ayuntamiento, haciendo entrega del hallazgo al señor Alcalde, en presencia de varios concejales y del oficial mayor...".

Este relato, de gran importancia, nos revela las circunstancias en las que se produjo el hallazgo, el día I I de enero de la llamada diadema, la sortija con epigrafía y el colgante con forma de media luna, mientras que el resto de las piezas fueron halladas al día siguiente por el profesor que, en compañía de los niños, volvieron al lugar donde se encontraron las primeras piezas.

Junto con el informe del profesor relatando el hallazgo el Director del Museo de Jaén, Don Juan González Navarrete, añadió la relación de objetos que se habían encontrado días antes, mencionando los siguientes materiales:

- Diadema de oro, muy parecida a la de la Aliseda en cuanto a su forma de 0,213 ×0,045. Está compuesta por cinco zonas rectangulares y dos triangulares en sus extremos. En cada una de las cinco zonas rectangulares se encuentra una cápsula elíptica con pasta vítrea blanca, menos una que es azul.

- Dos posibles pendientes o colgantes, uno en forma de media luna y otro muy fragmentado.

- Seis rectángulos de oro con cápsulas centrales y pasta vítrea, posiblemente de una pulsera.
- Cuatro rosetones de oro, dos de ellos con cápsula redonda y pasta vítrea y los otros dos, uno sin pasta vítrea y otro muy fragmentado.

- Tres cuentas de metal dorado.

- Cinco cilindros de oro, al parecer de un collar.

- Cuatro monedas árabes de plata, algunas con agujeros.

- Cuatro sortijas, una con inscripción y otra con cápsula elíptica y piedra rehundida.

- Dos posibles rosetones.

- Dos posibles broches.

- Una aguja, posiblemente de fíbula.

- Una cinta de plata, en dos fragmentos; en uno de sus extremos, tiene un pequeño cascabel.

- Cascabel mediano con cadena.

- Tres trozos unidos de cadena trenzada.

- Doce cuentas de collar grandes, de diferentes materias.

- Cincuenta y una perlitas, algunas minúsculas, de las llamadas aljófar.

- Seis trocitos de materias indeterminadas.

- Un trocito de hueso y otro de cerámica vidriada.

- Un trozo de cerámica común.

El tesorillo, fue encontrado en un terreno situado junto a la fábrica de aceite perteneciente a la Cooperativa Nuestra Señora del Rosario de Charilla, donde se estaba excavando una gran balsa para depositar la jamila ${ }^{2}$, según había ordenado el Instituto Nacional para la Conservación de la Naturaleza, para evitar así la contaminación de los ríos.

\footnotetext{
2 Alpechín.
} 
Tras este hallazgo, la construcción de la balsa fue paralizada con el objetivo de realizar una excavación cuando la tierra estuviese seca. La paralización de las obras de la construcción de la balsa para su futura excavación tuvo como consecuencia que la cooperativa tuviese que verter la jamila al río, por lo que la Comisaría de Aguas multó en dos ocasiones a la cooperativa por el vertido a través del arroyo Juan Castillo, al cauce público del arroyo Guadalcotón en su margen derecho. Las multas ascendieron a la cantidad de 5.000 y 1.000 pesetas respectivamente.

Una vez presentado el informe de cómo se encontró el conjunto y de qué piezas constaba el Director General del Patrimonio artístico y Cultural pidió al Director del Museo de Jaén que se procediese a su valoración y tasación para poder así incoar el expediente de indemnización, cumpliéndose así el artículo $5^{\circ}$ de la Ley de 7 de Julio de 191 I, que indica "será propiedad del Estado, a partir de la promulgación de esta Ley, las antigüedades descubiertas casualmente en el subsuelo o encontradas al demoler antiguos edificios". Siendo el resultado de esta valoración el siguiente:

I. Una diadema de oro bajo compuesta por cinco zonas rectangulares y dos triangulares, conteniendo en las zonas rectangulares sendas cápsulas elípticas con pasta vítrea blanca y una azul ..........240.000 pts.

2. Posibles pendientes o restos de los mismos compuestos por media luna y colgante 1.000 pts.

3. Tres anillos, uno con inscripción 10.000 pts.

4. Seis cuarterones, posiblemente de una pulsera 12.000 pts.

5. Cuatro rosetones y tres cuentas doradas... 10.000 pts.

6. Cuatro cuentas de collar, dos mayores y dos más pequeñas. 4.000 pts.

7. Tres monedas agujereadas árabes................2.400 pts.
8. Colección de piezas, seis de ellas grandes y el resto pequeñas.. 10.200 pts.

9. Una sortija de chatón sin piedra....................5.000 pts.

10. Dos cascabeles y una banda de plata ........2.000 pts.

1।. Tres cuentas cilíndricas metálicas, una moneda y una bolita dorada, todo en mala conservación. 2.400 pts.

12. Restos de huesos, cerámica y varios. 1.000 pts.

La valoración final del tesorillo fue de 300.000 pesetas correspondiendo la mitad de dicha cantidad a los tres alumnos y al profesor en calidad de descubridores, ya que, según el artículo $6^{\circ}$ de la Ley de 7 de Julio de 191। "el descubridor recibirá, al hacer entrega de los efectos encontrado, en ambos casos, como indemnización, la mitad del importe de la tasación legal de dichos objetos, correspondiendo la otra mitad, en el segundo caso, al dueño del terreno". Las I50.000 pesetas que correspondían tanto a los alumnos ${ }^{3}$ como al profesor fue pagada por el Estado el 21 de Junio de 1978, un año y medio después del descubrimiento, gracias a que el entonces Director del Museo Provincial del Bellas Artes de Jaén Juan González Navarrete agilizó los trámites, aunque nada pudo hacer para que la Cooperativa Nuestra Señora del Rosario, propietaria de los terrenos, cobrase la otra mitad de la indemnización, ya que a pesar de haber sido multada tras la paralización de las obras de la balsa y el posterior vertido de las jamilas al cauce público le fue denegado el derecho a percibir la mitad de la tasación legal por haberse considerado el hallazgo "casual" y no "al demoler antiguos edificios".

El conjunto, una vez que el Estado autorizó su adquisición, fue depositado por D. Juan González Navarrete el día 25 de marzo de 1977 en el Instituto de Conservación y Restauración de obras de Arte, para su posterior restauración, restauración que fue llevaba a cabo por Dña. Aurora Ma Mateu. En la copia del informe de ingreso de las piezas en el citado Instituto se puede observar cómo, con pos-

3 La indemnización fue cobrada por los padres de los alumnos, ya que ellos en esas fechas eran menores de edad. 
terioridad a la entrega de las piezas por parte del Director del Director del Museo Provincial de Bellas Artes de Jaén, fue depositada bajo el Número de Registro A-2958 la base de una cerámica que podría pertenecer a un arcaduz, y que pudo haber servido como contenedor del ocultamiento, y de la que hasta ahora no teníamos noticia.

El conjunto está formado por las siguientes piezas:

- Una diadema o ceñidor $\left(n^{\circ}\right.$ de inventario A-2932) (Fot. I), formada por una placa base compuesta por un cuerpo rectangular realizado en una fina lámina de oro de $14,6 \mathrm{~cm}$. de longitud por $3,6 \mathrm{~cm}$. de ancho, coronada en uno de sus extremos más largos por 16 triángulos de $1 \mathrm{~cm}$. de alto cada uno. A esta placa base se le une mediante soldadura a cada uno de sus extremos sendas placas pentagonales de $3,5 \mathrm{~cm}$. cada una, rematadas en el vértice por una argolla soldada en la parte posterior de $1 \mathrm{~cm}$. de diámetro. La placa base ha sido repujada con motivos vegetales, tal y como se puede ver en la parte delantera de la misma. El cuerpo central rectangular está dividido en cinco rectángulos iguales coronados por los 16 triángulos unidos a él por una de las bases, decorándose las otras dos aristas con hilo torso simple que discurre por la parte interior de las mismas y que acaban en su extremo más cercano a la base en forma circular. Cada uno de los cinco rectángulos tiene un cabujón central ovalado que contiene pasta vítrea de diferentes colores, rodeados todos por hilo torso simple. El cabujón se encuentra enmarcado por cuatro esferas soldadas, una a cada lado, rodeado a su vez en tres de los lados del rectángulo por una banda de esferas soldadas, rodeadas a su vez por hilo torso simple. El extremo del rectángulo que no tiene esta banda de esferas es la parte más distante a los 16 triángulos y en él y a la altura del cabujón hay un círculo rodeado por un hilo torso simple en cuyo interior hay un pequeño agujero, quizás para colgar de él algún otro motivo ornamental. Cada una de las cinco placas rectangulares está encuadrada por un hilo torso simple, al que hay que añadir otra banda realizada con el mismo motivo y que rodea todo el cuerpo central, dando la sensación de tratarse de un hilo torso doble y no de dos simples que es de lo que se trata en realidad. Las dos placas de los extremos tienen sus perímetros decorados con hilos torsos dobles, excepto en la base que se une al cuerpo central mediante una decoración ondulada, formando ésta a su vez la base de otra decoración triangular en cuyo interior hay un círculo que alberga una esfera igual que las anteriores.

- Un colgante en forma de media luna $\left(n^{\circ}\right.$ de registro A-2933) está realizado sobre una placa de 3 por $2,5 \mathrm{~cm}$. Tiene forma de media luna con las puntas hacia abajo repujada en la parte posterior con líneas diagonales en las que aparecen sobre impresionados unos círculos, que son el resultado de la decoración circular de la parte delantera del colgante. En la parte central aparece un cabujón con forma rectangular que albergaría en su interior pasta vítrea que no ha llegado hasta nosotros y que divide la composición en dos mitades. El cabujón aparece rodeado por hilo torso simple. Todo esto aparece a su vez inscrito en un círculo de hilo torso simple fino. A ambos lados del cabujón hay dos círculos de tamaño medio, uno a cada lado, realizados por hilo torso simple y en cuyo interior hay una gran esfera soldada sobre la que hay tres esferitas soldadas formando un triángulo. Entre estos dos círculos y el cabujón central hay otros cuatro círculos, dos a cada lado, formados por hilo torso simple y en cuyo interior hay una pequeña esfera soldada rodeada por otras más pequeñas soldadas a la placa base colocadas de forma aleatoria, habiendo dos en el lado derecho y cuatro en el izquierdo. En los dos extremos de la media luna hay otras dos pequeñas esferas estando una de ellas rodeada por hilo torso simple. Todo el colgante está rodeado por hilo torso doble, colocado antes de realizar la decoración interior, ya que algunas de las esferas están soldadas encima del hilo torso. En cada uno de los vértices del colgante hay 
una pequeña perforación de la cual colgaría algún otro ornamento. La argolla está formada por una lámina decorada con tres estrías doblada y soldada hacia la parte posterior de colgante.

- El llamado cinturón (A-2935) (Fot. 2) está compuesto por seis placas rectangulares independientes, iguales, de 1,8 por 2 centímetros, unidas entre sí mediante tres hilos que discurrirían por el interior de las piezas. Cada una de las piezas está compuesta por dos placas, una lisa en la parte inferior y otra decorada en la superior. La placa superior tiene un cabujón central ovalado en la que se insertó la pasta vítrea de colores, todo ello rodeado por hilo torso simple finísimo. El cabujón se encuentra enmarcado por cuatro esferas soldadas, una a cada lado, rodeado a su vez por una banda de esferas soldadas que discurren por los cuatro lados del rectángulo. Las dos placas están unidas entre sí por cuatro finas láminas, una a cada lado. Las dos de los extremos mayores presentan tres aberturas ovaladas en cada una de sus lados, aberturas rodeadas por hilo simple por la cual pasarían los hilos que unirían las seis piezas.

- Cinco brácteas, de las cuales tres están completas y dos incompletas. Dos de las brácteas completas, están formadas por diez lóbulos circulares (A-2936 y A-2938) en cuyo interior hay una esfera soldada y rodeada por un anillo de hilo simple. Entre cada uno de los lóbulos hay un círculo formado por hilo simple de menor tamaño que los anteriores. En la zona central hay otro anillo circular formado por hilo simple, en cuyo interior se eleva la corona de engaste donde se insertó la pasta vítrea, perdido en uno de los casos. La parte trasera de las dos placas base tiene decoración de grecas.

La tercera bráctea completa (A-2939), de menor tamaño que las anteriores, también tiene forma circular y está formada por diez lóbulos, aunque, a diferencia de las anteriores, no posee círculos de hilo torso entre ellos, ni tiene decorada la placa base. Lo que sí posee es una corona de engaste cir- cular en el centro de la bráctea, en cuyo interior encontramos pasta vítrea de color amarillento. A ambos lados de la bráctea encontramos dos parejas de argollas a modo de pasador que servirían para unir esta pieza a alguna prenda de vestir. De las dos brácteas incompletas, una de ellas está compuesta por un cabujón central circular que albergaría la pasta vítrea hoy desaparecida (A-2934), rodeado por hilo torso simple, en la actualidad sólo se conservan cuatro lóbulos circulares rodeados por hilo torso simple, en cuyo interior hay una esfera hueca. También se observan dos pequeñas argollas, una a cada lado de los lóbulos, formadas por un hilo plano colocado a modo de lazo y soldado a la parte posterior de la misma. Si comparamos esto con la parte perdida de la bráctea tendrían que ir otras dos argollas colocadas en al lado opuesto, tal y como podemos ver en una de las brácteas completas. Mientras que, de la otra bráctea circular, se conservan nueve lóbulos (A2937), a los que habrían que añadir otros dos desaparecidos en la actualidad. Los lóbulos están formados por una esfera circular gallonada, en cuya parte superior hay cuatro microesferas, rodeada por un anillo de hilo torso simple. Entre cada uno de los lóbulos hay una pequeña circunferencia formada también por hilo torso simple. En la parte que rodea el cabujón central de la bráctea hay dos círculos concéntricos realizados por hilo torso simple entre los cuales hay otros dos pequeños anillos realizados con el mismo motivo decorativo. En la zona central de la composición hay un cabujón que hoy se encuentra vacío. La placa base posterior es lisa.

- Tres cuentas de metal dorado de 1,5 cm. cada una. Las tres están huecas y tienen dos aberturas laterales, una a cada lado, siendo un orificio de entrada y otro de salida, por el cual se introduciría el hilo para engarzarlas. Estas aberturas están rodeadas por pequeñas microesferas soldadas a modo de decoración.

- Cinco canutos de oro, unos completos y otros incompletos (A-294I). Hay dos mode- 
los diferentes, uno de ellos está formado por un cuerpo central con diferentes perforaciones que siguen un orden lineal. Es una placa rectangular cerrada hacia dentro formando un canuto y soldado en el extremo. A cada uno de los lados hay un cuerpo lateral con forma de media esfera hueca rodeada por hilo torso simple. Hay un orificio rodeado también por hilo torso por el cual se introduciría el hilo de engarce. El otro de los modelos también está formado por un cuerpo central formado por medias microesferas huecas unidas entre ellas por un pequeño engarce rectangular en la parte trasera. Los cuerpos laterales tienen una decoración gallonada rodeada por un reborde de microesferas.

- Cuatro dirhemes perforados (A-2942), cuatro de Abd al-Rahman III, uno del 33I H./942 d.C. (Vives 397) y dos del 334 H./945 d.C. (Vives 408) de la ceca al-Andalus y uno del año i337 H./948? d.C. (Vives 46I), de Madinat al-Zahra.

- Cuatro anillos, uno de oro y tres de plata. El anillo de oro (A-2945) está formado por un aro de 1,95 cm. de diámetro y un chatón con un cabujón central ovalado en el que se ha colocado la pasta vítrea, y está rodeado por hilo torso simple, al igual que en la parte superior. Entre estas dos bandas y a cada uno de los cuatro lados hay un adorno formado por una especie de argolla. A ambos lados del cabujón y en el aro hay un motivo decorativo en forma de triángulo invertido realizado por pequeñas microesferas soldadas. El aro no es redondeado, sino que la parte exterior está ligeramente apuntada hacia fuera. El primero de los anillos de plata (A-2943) está formado por un aro de 1,7 cm. de diámetro al que hay unido una placa de forma cuadrangular de 1,2 $\times 0,5 \mathrm{~cm}$. con aristas curvas y una inscripción en su interior con el nombre de "Said". El segundo de los anillos de plata (A2944) tiene un aro de $2 \mathrm{~cm}$. de diámetro con un cabujón central ovalado de 1,7 × 1,4 $\mathrm{cm}$. en cuyo interior iría la pasta vítrea que no ha llegado hasta nosotros. Y el último de los anillos (A-2946), posee un aro de I,8 $\mathrm{cm}$. de diámetro y una placa rectangular lisa de $1,5 \times 1 \mathrm{~cm}$.

- Cuenta cilíndrica (A-2947-48), con forma de esfera hueca, decorada con círculos concéntricos.

- Dos elementos del cuerpo exterior de un canuto, el perteneciente al n ${ }^{\circ} \mathrm{A}-2949$, posee una base circular rodeada por hilo torso simple. La parte central está más elevada y perforada en el centro, por donde pasaría el hilo de engarce. Mientras que, el perteneciente al A-2950, es igual al anterior, pero con una decoración de hilo torso simple que rodea la abertura.

- Aguja (A-295I), se trata de una barra de plata redondeada de $5 \mathrm{~cm}$. de longitud, acabando uno de sus extremos en punta, mientras que, el otro está fragmentado.

- Cinta de plata (A-2952) fragmentada en dos, en uno de los extremos hay un cascabel.

- Cascabel con cadena (A-2953) realizado en dos mitades y unidos por un anillo. La cadena se une al cascabel mediante una argolla.

- Tres fragmentos de cadena (A-2954) unidos por una anilla de $1 \mathrm{~cm}$. de diámetro. Las tres cadenas miden $1,5 \mathrm{~cm}$., $5,2 \mathrm{~cm}$. y $3,2 \mathrm{~cm}$. respectivamente, y están formadas por eslabones dobles.

- Trece cuentas de collar (A-2956) formadas por piedras de diferentes colores perforadas en el centro, por cuyo interior discurre el hilo de engarce.

- Cincuenta y una perlas de aljófar (A-2956) perforadas para pasar un hilo de engarce.

- Cinco fragmentos diversos (A-2957), indeterminados.

- Fragmento de cerámica (A-2958), base de un posible arcaduz, que entró en Instituto de Conservación y Restauración de obras de Arte días después que el resto del conjunto, y del que sólo conservamos una fotografía (Fot. 3). 
La importancia de este conjunto no sólo radica en los elementos de joyería de los que se compone, sino en las cuatro monedas, ya que gracias a ellas se ha podido fechar con exactitud. En la actualidad se conocen cinco conjuntos de joyería califal aparecidos junto con material numismático — Conjunto de Charilla, de "Ermita Nueva", del Cortijo de "la Mora", de Loja y de Lorca-, más otros cuatro sin ellas - conjunto de Garrucha, Sierra Leones, elementos del Museo Arqueológico Nacional y el conjunto de The Walter Art Gallery-, con dudas sobre la cronología de este último que estilística y técnicamente difiere en gran medida de los fechados.

La cronología de las cuatro monedas que aparecieron en el Conjunto de Charilla -cuatro dirhemes de Abd al-Rahman III (una del año 33IH./942 d.C., dos del 334H./945d.C.) y una del año i337 H./948? d.C. de Madina al-Zahra están en consonancia con las aparecidas en el resto de los conjuntos, ya de los ciento quince dirhemes aparecidos en el conjunto de "Ermita Nueva" (Alcalá la Real, Jaén) cuarenta y dos eran de Abd al-Rahman III de los años comprendidos entre el 326H./937 d.C. al 350H./ 96 Id.C. de las cecas de al-Andalus y Madina al-Zahra; trece de Al-Hakam II de la ceca de Madina al-Zahra entre los años $350 \mathrm{H}-364 \mathrm{H} . /$ 96I-974 d.C., cuarenta y nueve de Hisam II entre los años 366H./ 976d.C. y 398H./ 1007 d.C. de las cecas de Madinat Fas y al-Andalus, tres monedas de Muhammad II de la ceca de al-Andalus del año 400H./ 009 d.C., cuatro de Sulayman del 400H.//009 d.C., de la ceca alAndalus, dos del $2^{\circ}$ reinado de Hisam II del 40 I H./I 010 d.C., de la ceca de al-Andalus, más dos monedas fatimíes del norte de África, a nombre del califa al-Hakam. Mientras que, los ochenta y seis dirhemes encontrados junto al Conjunto de Cortijo de la Mora (Lucena, Córdoba) van entre los años 325-39 I H./936- I000 d.C., perteneciendo a Abd al-Rahman III, de las cecas de Al-Andalus y Madina al-Zahra, Al Hakam II, de Madina al Zahra y Hixem II de Al-Andalus y Madinat Fas. De los seis dirhemes del Conjunto de Loja pertenecía dos son de Abd al-Rahman III de las cecas de Al-Andalus y Madina al-Zahra, dos de Hisam II de Al-Andalus, uno de Sulayman al-Mustacin de Al-Andalus y uno del fatimí al-Mucizz Abu Tamin Ma`ad. A lo que hay que añadir el lote de dirhemes citado por Gómez Moreno y cuya cronología más reciente es del 400H./I009 d.C. pertenecientes al conjunto de Lorca.

La comparación de estos conjuntos fechados con material numismático nos lleva a corroborar la cronología de los conjuntos que aparecieron sin él, ya que las similitudes tanto técnicas como estilísticas son evidentes.

El conjunto de Charilla guarda una clara similitud con cinco de los conjuntos de los que tenemos conocimiento hasta hoy, destacando el gran parecido que mantiene con dos de ellos, el de "Ermita Nueva", encontrado en las proximidades del de Charilla y el de Loja, hallado en Granada. El Conjunto de "Ermita Nueva" posee el mismo tipo de brácteas polilobuladas, que están presentes, también, en los conjuntos de Loja y Sierra Leones (Priego, Córboba). Asimismo en "Ermita Nueva" encontramos piedras de colores para engarzar, tutes y anillos. Resulta más llamativa la asombrosa similitud entre las seis plaquitas rectangulares, perteneciente, a un posible un cinturón, de Charilla y Loja, elementos que no se han encontrado en ninguno de los otros conjuntos, coincidiendo tanto en la técnica de fabricación, tamaño, como en el número de elementos. Este conjunto también está formado por tutes y canutos gallonados parecidos a los de Charilla y a los descontextualizados que encontramos en el Museo Arqueológico Nacional, elementos acompañados por una serie de anillos pertenecientes a un hombre, tal y como sucede con los hallados en Charilla.

Queda así patente la importancia de este conjunto dentro de la joyería califal, ya que gracias a sus piezas de joyería y monedas ayuda a precisar la cronología de alguno de los otros conjuntos que no poseen material numismático. 


\section{BIBLIOGRAFÍA}

CHICHARRO, J. L. (2000): España Año 1000, Año 2000. Madrid.

CHICHARRO, J.L. (200I): "El tesoro de Charilla". E esplendor de los Omeyas cordobeses. Granada, p.22l.

FORTEZA DEL REY, C. Y AGUSTÍ, E. ( 1998): “El tesorillo islámico de Garrucha del Instituto Valencia de Don Juan (Madrid)", Axarquia, 3, Almería, pp. 82-88.

GOMEZ MORENO, M. (195I): Arte árabe español hasta los almohades. Arte mozárabe. Ars Hispaniae, Vol 3, Madrid.

IDEAL, Diario Regional de Andalucia Oriental, N 13.739 , | |-2-1977, Sección La Región, pp. 12.

JAÉN, Diario Provincial del Movimiento, No I I.475, 9-21977, Sección Provincial, pp. 12.

JAÉN, Diario Provincial del Movimiento, No | I.523, 6-41977, Sección Provincial, pp. 16

LUNA, MåD. (1993): "Instrumental metálico de época hispanomusulmana en el Museo Histórico Municipal de Priego de Córdoba", Antiquitas, 4, Priego de Córdoba.
MAKARIOU, S. (200I): Les Andalousies: de Damas à Cordue. Institut du Monde Arabe. París, pp.68.

TORRES BALBAS, L. (1963): Arte hispanomusulmán hasta la caida del califato de Córdoba. $H^{a}$ de España de Ramón Menéndez Pidal, Tomo V, Madrid, pp. 729-730, 766-769.

VIVES ESCUDERO, A. (1893): Monedas de las dinastías arábigo-españolas, Madrid.

W.AA. (1997): Moneda Andalusí en la Alhambra, Catálogo de la Exposición, Sevilla, pp. 191-197.

W.AA. (1997): Santiago, Al-Andalus. Diálogos artísticos para un milenio. Vida y cultura en la ciudad hispano musulmana, pp. 247-269.

W.AA. (1998): Museo Histórico Municipal de Priego de Córdoba. Catálogo de la Exposición Conmemorativa de XV aniversario de creación. Córdoba, pp.45-46.

ZOZAYA, J. (1995): "El tesoro de Charilla". El zoco, vida económica y artes tradicionales en Al-Andalus y Marruecos. Barcelona, pp. 149.

ZOZAYA, J. (1997): "Diadema o ceñidor del Tesoro de Charilla". Al-Andalus: las artes islámicas en España. Madrid, pp. 205-206 


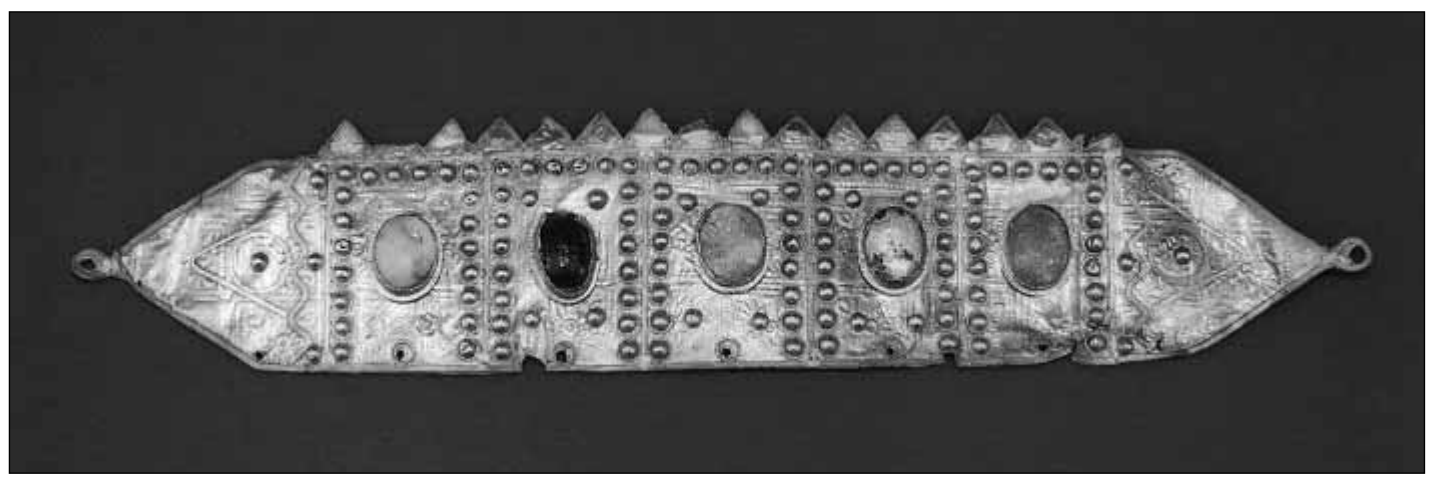

Fig. I. La llamada "diadema" o ceñidor del conjunto de Charilla.

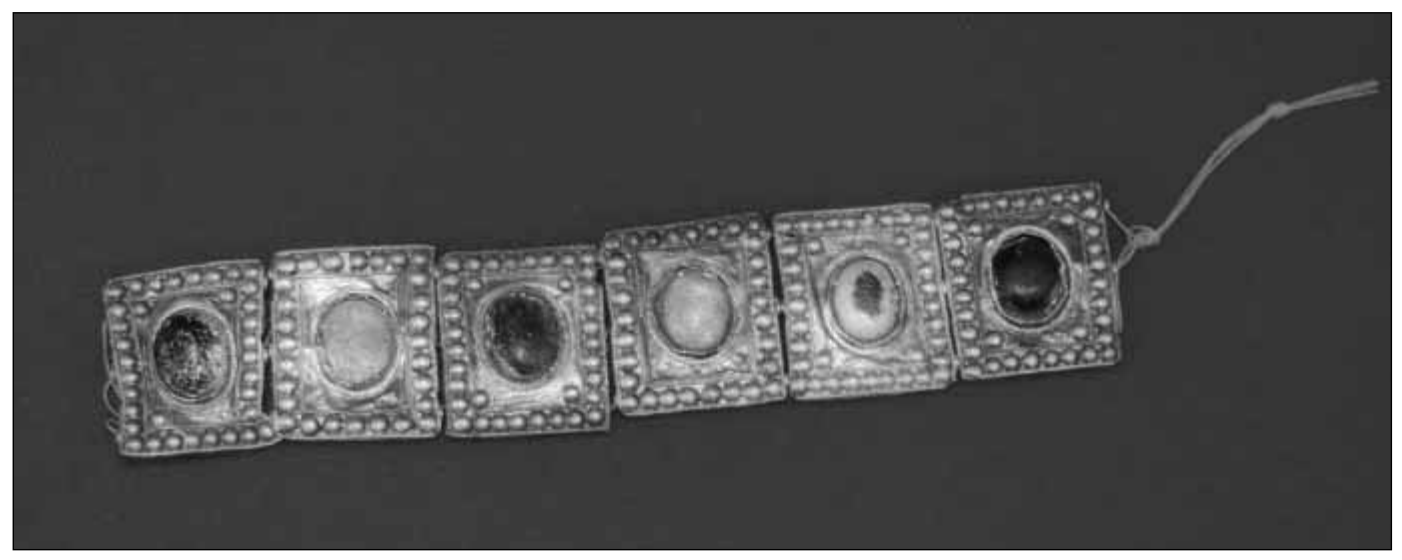

Fig. 2. Seis plaquitas del conjunto de Charilla consideradas como parte de un cinturón.

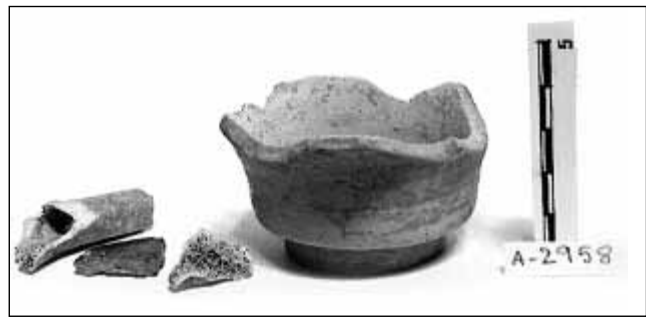

Fig. 3. Fragmento de arcaduz aparecido junto al conjunto de Charilla y que posiblemente fue el contenedor del mismo. (Proc. IPHE). 